\title{
Moderate-to-High Intensity Physical Exercise in Patients with Alzheimer's Disease: A Randomized Controlled Trial
}

\author{
Kristine Hoffmann ${ }^{\mathrm{a}}$, Nanna A. Sobol ${ }^{\mathrm{b}, \mathrm{c}}$, Kristian S. Frederiksen ${ }^{\mathrm{a}}$, Nina Beyer ${ }^{\mathrm{b}, \mathrm{c}}$, Asmus Vogel ${ }^{\mathrm{a}}$, \\ Karsten Vestergaard ${ }^{\mathrm{d}}$, Hans Brændgaard ${ }^{\mathrm{e}}$, Hanne Gottrup ${ }^{\mathrm{e}}$, Annette Lolk ${ }^{\mathrm{f}}$, Lene Wermuth ${ }^{\mathrm{f}}$, \\ Søren Jacobsen ${ }^{\mathrm{g}}$, Lars P. Laugesen ${ }^{\mathrm{h}}$, Robert G. Gergelyffy ${ }^{\mathrm{h}}$, Peter Høgh ${ }^{\mathrm{i}}$, Eva Bjerregaard', \\ Birgitte B. Andersen ${ }^{\mathrm{a}}$, Volkert Siersma ${ }^{\mathrm{k}}$, Peter Johannsen ${ }^{\mathrm{a}}$, Carl W. Cotman ${ }^{\mathrm{l}}$, Gunhild Waldemar ${ }^{\mathrm{a}}$ \\ and Steen G. Hasselbalch ${ }^{\mathrm{a}, *}$ \\ ${ }^{a}$ Danish Dementia Research Centre, Department of Neurology, Rigshospitalet, Copenhagen University Hospital, \\ Copenhagen, Denmark \\ ${ }^{\mathrm{b}}$ Musculoskeletal Rehabilitation Research Unit, University of Copenhagen, Denmark \\ ${ }^{\mathrm{c}}$ Institute of Sports Medicine Copenhagen, Bispebjerg Hospital, University of Copenhagen, Denmark \\ d Memory Clinic, Aalborg University Hospital, Denmark \\ e Dementia Clinic, Aarhus University Hospital, Denmark \\ ${ }^{\mathrm{f}}$ Dementia Clinic, Odense University Hospital, Denmark \\ ${ }^{\mathrm{g}}$ Department of Geriatrics, Odense University Hospital, Svendborg Hospital, Denmark \\ ${ }^{\mathrm{h}}$ Department of Geriatrics, Slagelse Hospital, Denmark \\ ${ }^{\mathrm{i}}$ Regional Dementia Research Center, Region Zealand, Roskilde Hospital, University of Copenhagen, Denmark

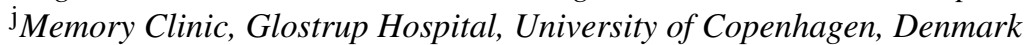 \\ ${ }^{\mathrm{k}}$ Research Unit for General Practice and Section of General Practice, Department of Public Health, \\ University of Copenhagen, Denmark \\ ${ }^{1}$ Institute for Memory Impairments and Neurological Disorders, University of California-Irvine, CA, USA
}

Accepted 21 October 2015

\begin{abstract}
.
Background: Studies of physical exercise in patients with Alzheimer's disease (AD) are few and results have been inconsistent. Objective: To assess the effects of a moderate-to-high intensity aerobic exercise program in patients with mild AD.

Methods: In a randomized controlled trial, we recruited 200 patients with mild AD to a supervised exercise group (60-min sessions three times a week for 16 weeks) or to a control group. Primary outcome was changed from baseline in cognitive performance estimated by Symbol Digit Modalities Test (SDMT) in the intention-to-treat (ITT) group. Secondary outcomes included changes in quality of life, ability to perform activities of daily living, and in neuropsychiatric and depressive symptoms. Results: The ITT analysis showed no significant differences between intervention and control groups in change from baseline of SDMT, other cognitive tests, quality of life, or activities of daily living. The change from baseline in Neuropsychiatric Inventory differed significantly in favor of the intervention group (mean: $-3.5,95 \%$ confidence interval (CI) -5.8 to $-1.3, p=0.002$ ). In subjects who adhered to the protocol, we found a significant effect on change from baseline in SDMT as compared with the control group (mean: $4.2,95 \%$ CI 0.5 to $7.9, p=0.028$ ), suggesting a dose-response relationship between exercise and cognition.
\end{abstract}

\footnotetext{
*Correspondence to: Professor, DMSc, MD, Steen G. Hasselbalch, Danish Dementia Research Centre, Section 6922, Department of Neurology, Rigshospitalet, University of
}

Copenhagen, 9 Blegdamsvej, DK 2100 Copenhagen, Denmark. Tel.: +45 45356702; E-mail: steen.gregers.hasselbalch@regionh.dk. 
Conclusions: This is the first randomized controlled trial with supervised moderate-to-high intensity exercise in patients with mild AD. Exercise reduced neuropsychiatric symptoms in patients with mild AD, with possible additional benefits of preserved cognition in a subgroup of patients exercising with high attendance and intensity.

Keywords: Alzheimer's disease, behavioral symptoms, cognition, exercise, randomized controlled trial

\section{INTRODUCTION}

The number of people living with Alzheimer's disease $(\mathrm{AD})$ is growing due to increasing life expectancy. The effect of pharmacological treatment is limited, and no new drugs have been approved in the last decade [1]. There is an urgent need for alternative treatments, including non-pharmacological approaches.

Various longitudinal cohort studies have suggested that physical activity in middle age protects against cognitive decline and dementia in old age $[2,3]$ and preserves the ability to perform activities of daily living (ADL) among older healthy subjects [4]. Moreover, a dose-response has been suggested in healthy subjects showing that higher levels of physical activity are associated with better cognitive performance [5]. However, a recent large randomized trial in sedentary adults failed to show any effect on cognitive outcomes after 24 months of moderate intensity physical exercise [6].

Almost all randomized studies in patients with mild cognitive impairment (MCI) demonstrate some positive effects on one or several domains of cognition [7]. In one of the largest randomized trials of aerobic exercise in MCI subjects, tests of executive function (Symbol Digit Modalities, Verbal Fluency, and Stroop) showed improvement, whereas memory tests did not, suggesting that certain cognitive domains are more amendable to exercise than others [8]. In studies of dementia and $\mathrm{AD}$, one recent review [7] and an updated meta-analysis [9] underscore the need for caution in interpreting findings due to methodological issues. Further, some studies in patients with AD or MCI have relied on unsupervised, caregiver supervised, or partly unsupervised exercise $[8,10,11]$. Problems with small samples sizes, inadequate randomization procedures, and a lack of sufficient measures of exercise intensity also underscore the need for more rigorously conducted research in this area [7].

Although exercise may yield benefits in quality of life and neuropsychiatric and depressive symptoms in patients with $\mathrm{AD}$, a recent meta-analysis concluded that there was not much evidence for such an effect [9]. None of the studies on neuropsychiatric symptoms involved community-dwelling patients, which is a critical issue, as these symptoms are the primary cause of caregiver burden and later nursing home placement [12].

To our knowledge this is the first study to investigate the potential benefits of continuously supervised moderate-to-high intensity aerobic exercise on cognition, neuropsychiatric and depressive symptoms, health-related quality of life and ADL, in communitydwelling patients with mild AD.

\section{MATERIAL AND METHODS}

\section{Study design}

The Preserving Cognition, Quality of Life, Physical Health and Functional Ability in Alzheimer's Disease: The Effect of Physical Exercise (ADEX) study was a multi-center, single-blind, randomized trial conducted between January 2012 and June 2014. Participants were randomized to a control group, which received treatment as usual, or an intervention group, which performed $60 \mathrm{~min}$ of supervised moderate-tohigh intensity aerobic exercise three times weekly for 16 weeks. An uncontrolled pilot study was carried out in 2011 [13] to ensure the acceptability, safety, and feasibility of the intervention, as recommended in the Medical Research Council's guidelines for complex interventions [14]. A detailed description of the methods of the study has previously been published [15].

\section{Ethical approval}

The trial protocol was approved by the Danish National Committee on Biomedical Research Ethics (H-3-2011-128) and written informed consent was obtained from all participants. The Danish Data Protection Agency approved the research database (ID No 30-0718).

\section{Study participants}

Two-hundred community-dwelling patients with mild AD were included. All patients had a clinical diagnosis of probable $\mathrm{AD}$ according to the NINDS- 
ADRDA Alzheimer's Criteria [16]. Subjects were recruited from eight outpatient memory clinics in Denmark. All sites listed in the acknowledgment participated in the data collection.

Inclusion criteria included a Mini Mental State Examination (MMSE) score $>19$ (at screening less than six weeks prior to baseline visit), age between 50-90 years, and a caregiver with regular contact (more than once a month) who was willing to participate in the study. If patients received anti-dementia medication or mood stabilizing medication, they had to be on a stable dose for at least three months before inclusion.

Exclusion criteria were 1) presence of cardiac or other medical diseases constituting a contraindication to physical activity or other neurological diseases causing cognitive decline (including severe cerebrovascular disease judged from cranial computed tomography or magnetic resonance imaging); 2) severe psychiatric disease; 3) alcohol abuse within the last two years according to the national guidelines; and 4) participation in regular physical activity of high intensity two or more times weekly. Detailed inclusion and exclusion criteria have previously been described [15].

\section{Study procedures}

Participants were included every six months over a period of 2.5 years. After screening for eligibility and obtaining informed consent, assessors (blinded to the group assignment throughout the study period) completed the baseline assessments. Subsequently, participants were randomized in blocks of 4-10 per participating center, using a computerized randomnumber generator. In the case of an unequal number of participants in one center, randomization was set up to favor the intervention group. Patients who discontinued participating in the intervention were encouraged to participate in the follow-up visit.

\section{Intervention group}

Three weekly exercise sessions were conducted in a group of 2-5 participants supervised by an experienced physiotherapist. The first four weeks of exercise (adaption) emphasized getting used to exercising and building up strength, primarily of the lower extremities (twice weekly). Participants were also introduced to aerobic exercise (once weekly). For the remaining 12 weeks, patients performed aerobic exercise of moderate-to-high intensity (in total $3 \times 10 \mathrm{~min}$ on an ergometer bicycle, cross trainer, and treadmill with 2-5 min rest between). Average heart rate (HR) was registered using continuous monitoring during aerobic exercise, including the rest intervals. The target intensity was $70-80 \%$ of maximal HR (220 - the person's age). To ensure that participants exercised with the intended intensity throughout the training period, average HR was further calculated for three time periods (between weeks 4 to 8,8 to 12 , and 12 to 16) as: (average HR of all sessions in a 4-week period)/(maximal HR) [17]. The training log also included information about training instruments and attendance. Attendance rate was defined as: (total number of attended training sessions)/(total number of offered training sessions).

\section{Control group}

Participants assigned to the control group received treatment as usual with access to memory clinic staff if medical or other needs necessitated contact during the study period. In order to increase adherence and positive expectations to the study, all control group subjects were offered 4 weeks of adaptation exercise after the termination of the study.

\section{Outcomes}

The primary outcome measure was the Symbol Digit Modalities Test (SDMT) [18], which assesses mental speed and attention. Using the number and symbol key at the top of the test page, participants were asked to correctly decode several lines of symbols. The total number of correct decodings in $120 \mathrm{~s}$ was used as outcome. Secondary outcome measures comprised of the Alzheimer's Disease Assessment Scale - Cognitive Subscale (ADAS-Cog) verbal memory test [19] (10 words recalled three times, immediate and delayed recall); the Stroop Color and Word Test (Stroop) [20] incongruent score; verbal fluency (number of words beginning with F, A, S, and animals named for $1 \mathrm{~min}$ each); and the MMSE [21]. The Hamilton Depression Rating Scale 17 items (HAMD-17) [22] was used to rate the severity of depressive symptoms.

Caregiver questionnaires included the Alzheimer's Disease Cooperative Study-Activities of Daily Living Inventory (ADCS-ADL) [23] scale and the 12-item Neuropsychiatric Inventory (NPI-12) [24].

The European Quality of Life-5 Dimensions (EQ5D) [25] health-related quality of life scale, which includes a visual analogue scale (EQ-5D VAS), was administered to both caregiver and patient [26].

All raters performing the outcome measurements were blinded to group assignment, and patients and caregivers were advised not to disclose group assignment during the test sessions. 


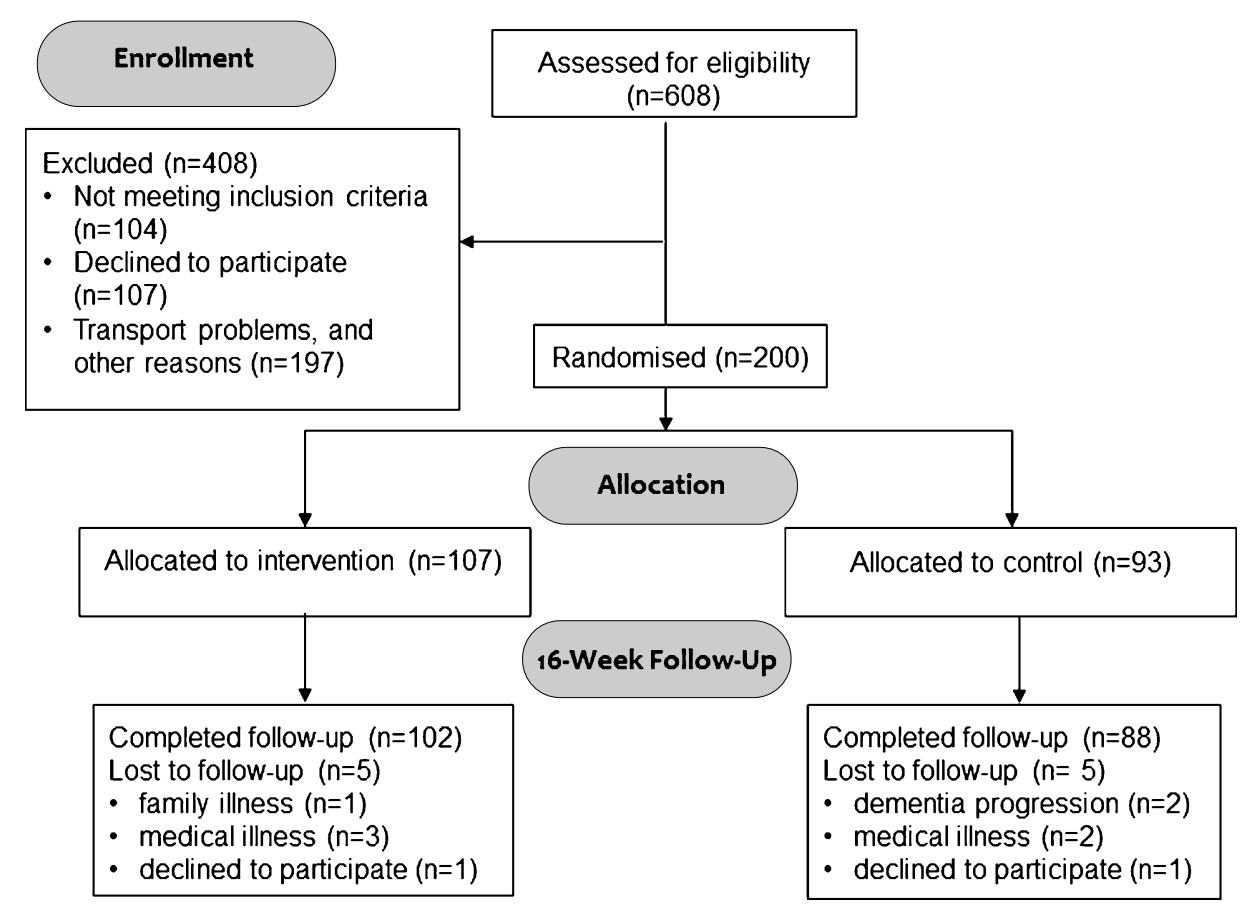

Fig. 1. Flow diagram of the ADEX study. Study participation and follow-up including reasons for drop-out.

\section{Statistical analysis}

Sociodemographic characteristics, baseline comorbidities, and medicine use, as well as the outcome measures at baseline, were compared between randomization groups using the Wilcoxon signed rank test for continuous variables and Fisher's exact tests for categorical variables.

The differences in change in outcomes from baseline to follow-up at 16 weeks between randomization groups were analyzed in linear regression models using generalized estimating equations to account for repeated measurement. Memory clinic and group indicator variables were included in the models to account for possible clustering by training groups. Missing data may have different causes for the two randomization groups and to avoid the associated bias, the measurements available at follow-up were weighted by the inverse of an estimate of the probability of still being in the study [27]. These probabilities were estimated from the data in a logistic regression model with the patient's baseline characteristics and the observed outcome at baseline as covariates. In the analysis of secondary outcomes, multiple comparisons were adjusted for by controlling the false discovery rate at 5\% [28].

In addition to intention-to-treat (ITT) analyses, we analyzed outcomes in a per protocol population (high exercise subjects), defined as participants in the inter- vention arm with a training attendance of more than $80 \%$ and average training intensity of more than $70 \%$ of maximal HR. The per protocol definition was set a priori based on previously accepted compliance thresholds applied in pharmaceutical trials [29] and on the lower limit of the intended intensity level. Because this analysis was exploratory, no correction for multiple comparisons was applied.

\section{RESULTS}

\section{Participant baseline characteristics}

Of the 608 individuals screened for eligibility, 200 were randomized and included in the ITT analysis (Fig. 1). There were no significant differences in baseline characteristics between the intervention group and the control group in any of the parameters (Table 1). One-hundred and thirteen were males (57\%), subjects had a mean age of 70.5 years, and a mean MMSE score of 24.0. A total of 190 (96\%) subjects completed follow-up. Eighty-one of 107 participants in the intervention group (76\%) attended more than $80 \%$ of the exercise sessions; 83 subjects $(78 \%)$ exercised with an intensity of more than $70 \%$ of maximal HR during the exercise sessions; and 66 subjects (62\%) fulfilled both criteria (high exercise subjects). There were no significant differences in baseline demographics 
Table 1

Baseline demographic and clinical characteristics of the study population

\begin{tabular}{|c|c|c|c|c|}
\hline \multirow[b]{2}{*}{$\mathrm{N}(\%)$} & \multirow{2}{*}{$\begin{array}{c}\text { Control } \\
93(46.5 \%)\end{array}$} & \multirow{2}{*}{$\begin{array}{l}\text { Intervention } \\
107(53.5 \%)\end{array}$} & \multicolumn{2}{|c|}{ Total } \\
\hline & & & $200(100 \%)$ & range \\
\hline \multicolumn{5}{|l|}{ 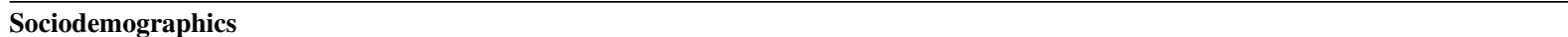 } \\
\hline \multicolumn{5}{|l|}{ Gender, $n(\%)$} \\
\hline Women & $36(38.7)$ & $51(47.7)$ & $87(43.5)$ & \\
\hline Men & $57(61.3)$ & $56(52.3)$ & $113(56.5)$ & \\
\hline Age (years), mean $(S D)$ & $71.3(7.3)$ & $69.8(7.4)$ & $70.5(7.4)$ & \\
\hline Caregiver living with patient, $n(\%)$ & $66(71.0)$ & $78(72.9)$ & $144(72.0)$ & \\
\hline Physical Activity Scale for the Elderly*, mean (SD) & $97.2(49.0)$ & $95.2(41.6)$ & $96.5(45.1)$ & \\
\hline \multicolumn{5}{|l|}{ Comorbidities } \\
\hline Hypertension, $n(\%)$ & $35(37.6)$ & $48(44.9)$ & $83(41.5)$ & \\
\hline Diabetes, $n(\%)$ & $7(7.5)$ & $11(10.3)$ & $18(9.0)$ & \\
\hline Hypercholesterolemia, $n(\%)$ & $34(36.6)$ & $40(37.4)$ & $74(37.0)$ & \\
\hline Stroke, $n(\%)$ & $2(2.2)$ & $1(0.9)$ & $3(1.5)$ & \\
\hline Acute myocardial infarction, $n(\%)$ & $1(1.1)$ & $2(1.9)$ & $3(1.5)$ & \\
\hline \multicolumn{5}{|l|}{ Medicine } \\
\hline Antihypertensive treatment, $n(\%)$ & $39(41.9)$ & $48(44.9)$ & $87(43.5)$ & \\
\hline Antidepressants, $n(\%)$ & $28(30.1)$ & $29(27.1)$ & $57(28.5)$ & \\
\hline Anti-Alzheimer's treatment, $n(\%)$ & $88(94.6)$ & $105(98.1)$ & $193(96.5)$ & \\
\hline Beta-blocker treatment, $n(\%)$ & $9(9.7)$ & $7(6.5)$ & $16(8.0)$ & \\
\hline Symbol Digit Modalities Test ${ }^{\S}$, mean (SD) & $25.4(14.3)$ & $27.1(14.7)$ & $26.7(14.5)$ & $0-63$ \\
\hline Mini Mental State Examination", mean (SD) & $24.1(3.8)$ & $23.8(3.4)$ & $24.0(3.6)$ & $14-30$ \\
\hline Hamilton Depression Rating Scale 17 items $^{\dagger}$, mean (SD) & $2.0(2.5)$ & $1.9(2.6)$ & $2.0(2,6)$ & $0-11$ \\
\hline Neuropsychiatric Inventory $\ddagger$, mean (SD) & $9.4(9.7)$ & $10.0(10.8)$ & $9.7(10.3)$ & $0-42$ \\
\hline
\end{tabular}

Fisher's exact test was used for categorical variables and Wilcoxon signed-rank test was used for continuous variables; there were no significant between-group differences in any baseline characteristics. *Physical activity was measured by means of the Physical Activity Scale for the Elderly [41] (derived by multiplying activity participation by established item weights). Higher scores indicate higher levels of habitual physical

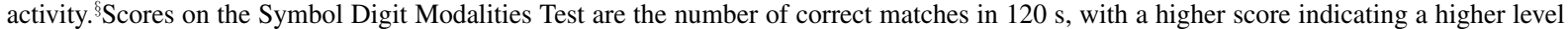

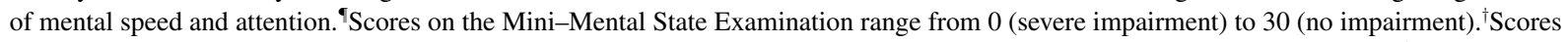
for the 17-item Hamilton Depression Rating Scale range from 0 to 52, with higher scores indicating more severe depression. ${ }^{\ddagger}$ Scores on the Neuropsychiatric Inventory range from 0 to 144, with higher scores indicating increased behavioral and psychological symptoms.

between the 3 groups (control, low, and high exercise, see Supplementary Table 1), however, the proportion of patients with arterial hypertension was greater in the low exercise group, and none of the patients treated with beta-blockers fulfilled the high exercise criteria as they could not reach the level of more than $70 \%$ of maximal HR during the exercise.

\section{ITT analyses}

Mean $( \pm \mathrm{SD})$ between-group differences in outcomes at follow-up are shown in Table 2. There was no significant difference in change from baseline to follow-up between groups on the primary outcome (SDMT), although there was a 2.5 point $(95 \% \mathrm{CI}-1.1$ to $6.1 ; p=0.179$ ) difference between the groups in favor of the intervention group. There was a significant difference in the change in total neuropsychiatric symptoms assessed by NPI by -3.5 points $(95 \%$ confidence interval $[\mathrm{CI}],-5.8$ to $-1.3 ; p=0.002$ ) from baseline to follow-up indicating less severe neuropsychiatric symptoms in the intervention group. The effect of physical exercise seemed to be driven by an effect on several of the sub-items of the NPI scale (Fig. 2). No other secondary outcomes showed significant between-group differences in the changes from baseline to follow-up (Table 2).

\section{Per protocol analyses}

At follow-up, high exercise subjects $(n=66)$ differed from the control group by 4.2 points on the SDMT (95\% CI, 0.5 to $7.9 ; p=0.028$ ) (Table 2). The effect of exercise on NPI remained significant in high exercise subjects, while no other significant effects were found on the other secondary outcomes in high exercise subjects relative to the control group. A trend toward a difference at follow-up in quality of life on the patientrated EQ-5D VAS was found in high exercise subjects relative to the control group ( 4.5 points, $95 \% \mathrm{CI},-0.8$ to $9.8 ; p=0.097$ ).

\section{Drop-out and safety}

Ten patients were lost to follow-up. Reasons for drop-out are listed in Fig. 1. In all, 58 adverse events 


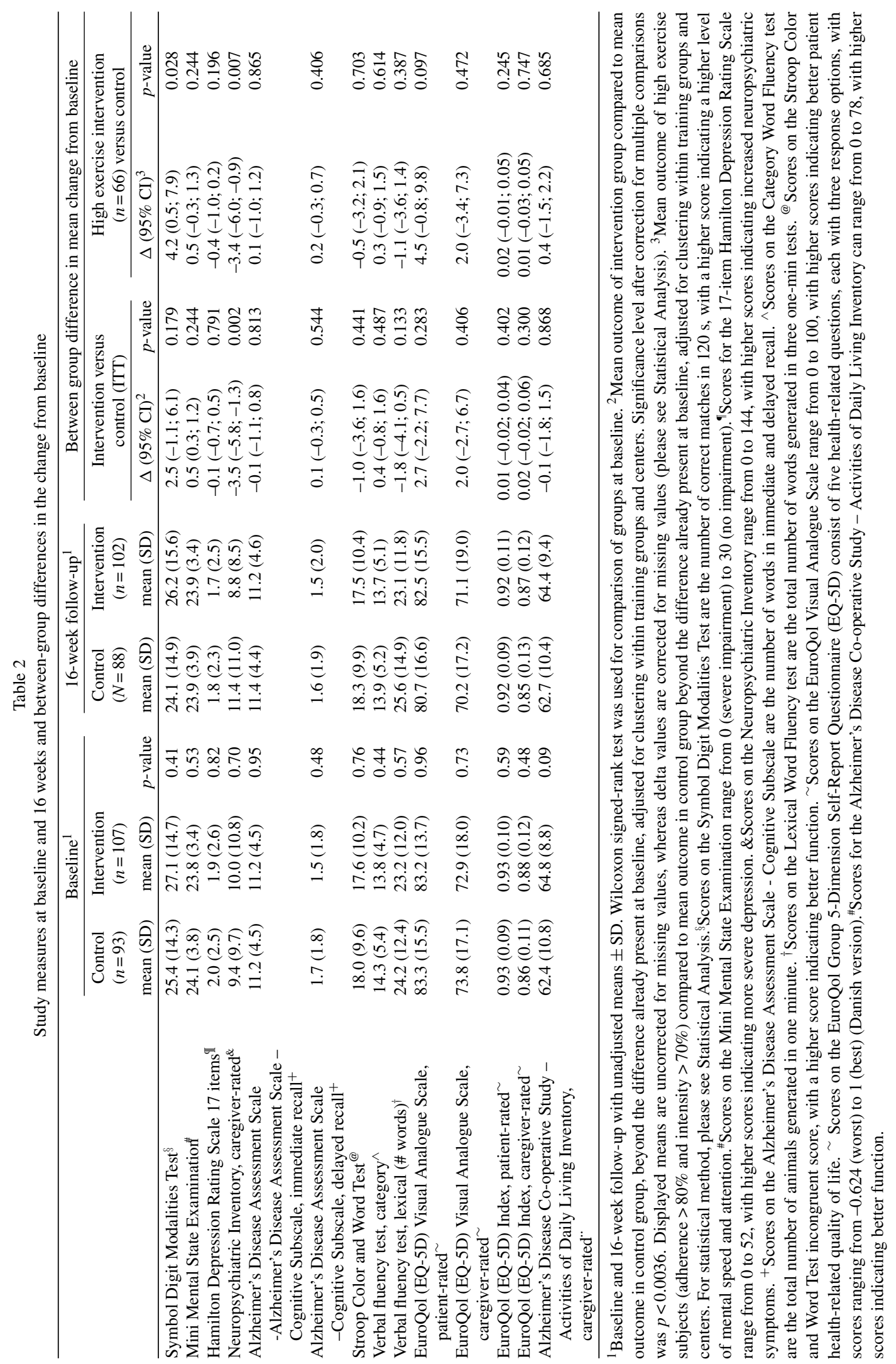




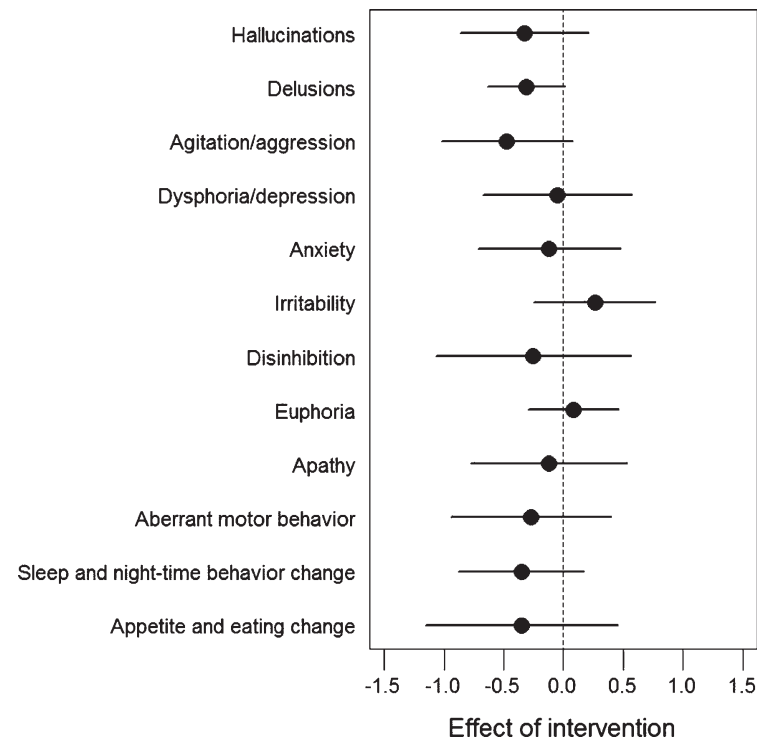

Fig. 2. Difference in changes in neuropsychiatric symptoms between intervention and control group. Between-Group Differences in the Change from Baseline on the 12 sub-items of the Neuropsychiatric Inventory (ITT analysis, mean values, bars denote 95\% CI). Negative values indicate less severe symptoms, and positive values indicate more severe symptoms after 16 weeks of exercise. No statistical analysis was applied, but the effect of exercise seemed to be driven by a general effect on several sub-items.

(AE) and 13 serious adverse events (SAE) were reported (Table 3). One incidence of atrial fibrillation was observed during a training session in a patient with no previous history of atrial fibrillation. Although the most likely scenario was that the increased monitoring during the training session revealed undiagnosed paroxysmal atrial fibrillation, it cannot be ruled out that the exercise triggered atrial fibrillation. The most common AE was musculoskeletal problems, with 16 events being reported, of which only 6 were related to the study.

\section{DISCUSSION}

This is the first rigorously conducted study of moderate-to-high intensity aerobic exercise in mild AD. Although the primary outcome was negative, there are several important findings in the study. Attendance was high (mean: 84\%) and in line with previous studies showing that aerobic exercise is a feasible and wellperceived intervention for older adults with AD [13, 30]. Following a supervised 16-week exercise program of moderate-to-high intensity, neuropsychiatric symptoms were significantly less severe. Although our primary outcome, SDMT, was negative in the ITT analysis, we found a possible effect on cognition in subjects adhering to the program. This could suggest that physical exercise may have an effect on cognition provided that high attendance and intensity is maintained. We did not find effects on measures of activities of daily living, depressive symptoms, or health-related quality of life. Serious adverse effects were rare and dropout rates were low, further indicating the safety and feasibility of this intervention in patients with mild AD.

The strengths of our study include a meticulous design, the large number of patients, the supervised exercise program, a high rate of adherence to the intervention, and the use of validated outcome measures. Weaknesses include lack of monitoring of the physical activity in the two groups outside the exercise sessions, the lack of a social activity program in the control group, an exploratory per protocol analysis that may have been underpowered to show statistically significant effects, and a selection bias which could mean that the intervention may not be applicable to all patients with mild AD.

Our findings of an effect of the intervention on neuropsychiatric symptoms are interesting, because a

Table 3

Adverse events (AE) and serious adverse events (SAE)

\begin{tabular}{|c|c|c|c|}
\hline & & Control & Intervention \\
\hline \multirow[t]{4}{*}{$\mathrm{AE} n=58$} & Musculoskeletal problems & 4 & 12 (6 related $)$ \\
\hline & Dizziness or faintness & 1 & 10 (6 related) \\
\hline & Symptoms related to $\mathrm{AD}$ & 4 & 3 \\
\hline & Somatic disease (i.e., cold, anemia, erysipelas) & 11 & 10 \\
\hline Total & & 20 & 35 \\
\hline \multirow[t]{7}{*}{ SAE $n=13$} & Pneumonia & 1 & 1 \\
\hline & Urinary tract infection & 1 & \\
\hline & Pulmonary edema & 1 & \\
\hline & Nephrolithiasis & 1 & \\
\hline & Atrial fibrillation & & $2(1 \text { possibly related })^{*}$ \\
\hline & Fracture & 2 & 3 \\
\hline & Breast cancer & & 1 \\
\hline Total & & 6 & 7 \\
\hline
\end{tabular}

Numbers of adverse effect and relation to study. ${ }^{*}$ One of the serious adverse events were possible related to the study, an atrial fibrillation event may have been triggered by the intervention. 
recent Cochrane Review concluded that exercise studies on neuropsychiatric symptoms, which were able to show a similar effect, were of insufficient quality [9] though, in that review, some previous low intensity or multimodal intervention studies were suggestive that exercise improved neuropsychiatric symptoms [31, 32]. Our findings indicate that physical exercise may inhibit or delay the emergence of more severe neuropsychiatric symptoms with disease progression. The difference between the intervention group and the control group tended to be explained by increasing severity of already present symptoms in the latter group, which did not occur in the intervention group. Although not explicitly tested, there seemed to be a general effect on neuropsychiatric symptoms, but our study was not designed to specifically address this issue. We did not find significant effects on depressive symptoms as measured by the Hamilton Depression Rating Scale, which could be due to the low prevalence of depressive symptoms at baseline in both groups. We were not able to test whether the effect on neuropsychiatric symptoms was mediated by the exercise itself, caregiver relief, positive expectations concerning the intervention, or by the social interaction between patients during group exercise. One could speculate that effects not directly related to exercise also would have affected other proxy-rated outcomes, such as quality of life and activities of daily living, which was not the case, but the study was not designed to differentiate between the different aspects of the complex intervention.

The negative findings on other secondary outcomes such as activities of daily living and quality of life may have several explanations. The relatively high mean baseline values on both scales (above 70 on proxy-rated EQ5D-VAS scale, and ADCS-ADL score of 62 out of 78 points) may have rendered these outcomes less sensitive to changes. Another possible explanation for the discrepant effects on the aforementioned outcomes and neuropsychiatric symptoms may be that the latter are more amenable to a short-term intervention, while an intervention of longer duration is required to affect quality of life and activities of daily living.

Our results indicated a dose-response of exercise on our primary outcome since the high exercise subjects showed less decline on SDMT than the control group in an exploratory analysis. A dose-dependent neuroprotective relationship between physical exercise and cognitive performance could in theory be mediated through reduction in cardiovascular risk factors [5] or other neuroprotective changes, including increases in the brain-derived neurotrophic factor and reductions in pro-inflammatory cytokines $[8,33,34]$. However, apart from the exploratory analysis of SDMT, the effect on cognition was largely negative in this study, and further studies are warranted before any claim for an effect of physical exercise on cognition in AD can be made.

Results have been conflicting with regards to which cognitive domains are most sensitive to exercise. SDMT was chosen as primary outcome because this test is sensitive to very early changes in aspects of executive function (mental speed and attention) in AD [35]. Further, executive function has been considered to be particularly sensitive to the effect of physical exercise $[36,37]$, which recently was corroborated in a large trial of moderate-intensity exercise in sedentary older adults where executive function was improved in the most cognitively impaired subgroup of subjects [6]. Lastly, in a rigorously conducted study in MCI patients involving moderate-to-high intensity aerobic exercise, the authors found a positive effect on this test [8]. Therefore, the very modest effect of exercise on cognitive function cannot in all likelihood be accounted for by the use of an insensitive measure.

The lack of effect on cognition in our ITT analyses may have several other reasons, including the lack of adherence to the exercise program in some patients, as suggested by our per protocol analysis. Although changes in cognitive function have been demonstrated in studies with small sample-sizes and different types of physical exercise, but with a duration similar to ours $[11,38,39]$, it has recently been suggested that at least six months of exercise is necessary to induce cognitive changes [40]. Thus, the duration of exercise period may be of importance, as suggested by a recent report [41]. As judged from the proxy-rated Physical Activity Scale for the Elderly [42], our patients were less physically active before entering the study as compared to healthy older adults [43], but probably more physically active than the general AD population due to selection bias. Including physically active patients in the study may have diminished the possibility of detecting the beneficial effects of the exercise program.

Furthermore, as we did not monitor the physical activity levels in the two groups outside the exercise sessions, we cannot rule out that changes in physical activity level other than the exercise intervention may have influenced outcome differences (i.e., participants in the intervention group being less physically active between exercise sessions or control group participants increasing their physical activity during the study).

The potential clinical relevance of our findings should be judged by the relative modest effect sizes. For a clinical relevant effect of 4 point or more on the SDMT, the number needed to treat would be 
25. In contrast, 8 patients would need treatment to obtain a clinical relevant improvement on NPI (arbitrarily defined as -4 point or less). The latter finding could be relevant as neuropsychiatric symptoms are associated with increased caregiver distress, increased admissions to nursing homes, and reduced quality of life in later stages of $\mathrm{AD}$ [12]. Because we selected patients able and willing to participate in an exercise program of moderate-to-high intensity, the results may not necessarily apply to the general AD population. We chose group exercise due to its cost-effectiveness and appropriateness for community-based programs. Interventions combining social interaction with specific training programs may be optimal, especially for diseases with complex symptomatology such as AD [44]. Furthermore, according to feed-back from participants, social interaction may at least explain part of the high adherence to the program.

Considering the potential effect, the few adverse events of the intervention, and the lack of efficient treatment strategies in $\mathrm{AD}$, there could be a potential for physical exercise as an add-on therapy in patients with AD. In particular, given the limited efficacy and high rate of adverse effects of psychotropic medication [45, 46], as well as the burden of neuropsychiatric symptoms [47], the current program could be considered as an add-on therapy in a community setting.

Future studies should clarify the potential dosedependent effect on cognition that we may have observed in the per protocol analysis. Trying to disentangle the effect of exercise from the effect of social interaction is another avenue for further study.

Moreover, studies are needed to confirm the findings from our study, and should also aim at further elucidating the underlying possible mechanisms of exercise. It should be clarified whether beneficial effects are symptomatic or disease modifying. Equally as important is to determine the optimal selection of type, dosage, and intensity of exercise.

In conclusion, our study shows that even a relatively short-term intervention can be effective in reducing neuropsychiatric symptoms of patients with mild AD. Furthermore, the data suggest that the dose of exercise impacts the effect on cognition.

\section{ACKNOWLEDGMENTS}

Professor, DMSc, MD Gunhild Waldemar and Professor, DMSc, MD Steen Hasselbalch, Danish Dementia Research Centre, Department of Neurology, Copenhagen University Hospital, Rigshospitalet, Denmark, are the ADEX study program director, and the national principal investigator, respectively, and Assoc. Professor Nina Beyer, Musculoskeletal Rehabilitation Research Unit and Institute of Sports Medicine Copenhagen, Copenhagen University Hospital, Bispebjerg, Denmark is the project director of physical exercise. We are grateful to all patients, caregivers, physiotherapists, study nurses, and clinical raters for their contributions to the ADEX study.

The ADEX study is supported by the Innovation Fund Denmark (J No. 10-092814). Additional funding was obtained by The Lundbeck Foundation (grant number FP 73/2012). The Danish Dementia Research Centre is supported by grants from the Danish Ministry of Health (J No. 2007-12143-112, project 59506/J No. 0901110, project 34501) and the Danish Health Foundation (J No. 2007B004). All researchers were independent from the funders, and the funders were not involved in the collection, analysis, and interpretation of data, in the writing of the report, or in the decision to submit the article for publication.

Authors' disclosures available online (http://j-alz. com/manuscript-disclosures/15-0817r1).

\section{SUPPLEMENTARY MATERIAL}

The supplementary material is available in the electronic version of this article: http://dx.doi.org/ 10.3233/JAD-150817.

\section{REFERENCES}

[1] Castellani RJ, Perry G (2012) Pathogenesis and diseasemodifying therapy in Alzheimer's disease: The flat line of progress. Arch Med Res 43, 694-698.

[2] Sofi F, Valecchi D, Bacci D, Abbate R, Gensini GF, Casini A, Macchi C (2011) Physical activity and risk of cognitive decline: A meta-analysis of prospective studies. J Intern Med 269, 107-117.

[3] Kulmala J, Solomon A, Kåreholt I, Ngandu T, Rantanen T, Laatikainen T, Soininen H, Tuomilehto J, Kivipelto M (2014) Association between mid- to late life physical fitness and dementia: Evidence from the CAIDE study. J Intern Med 276, 296-307.

[4] Shah RC, Buchman AS, Leurgans S, Boyle PA, Bennett DA (2012) Association of total daily physical activity with disability in community-dwelling older persons: A prospective cohort study. BMC Geriatr 12, 63.

[5] Weuve J, Kang JH, Manson JE, Breteler MM, Ware JH, Grodstein F (2004) Physical activity, including walking, and cognitive function in older women. JAMA 292, 1454-1461.

[6] Sink KM, Espeland MA, Castro CM, Church T, Cohen R, Dodson JA, Guralnik J, Hendrie HC, Jennings J, Katula J, Lopez OL, McDermott MM, Pahor M, Reid KF, Rushing J, Verghese J, Rapp S, Williamson JD, Study LIFE, Investigators (2015) Effect of a 24-month physical activity intervention vs health education on cognitive outcomes in sedentary older adults: The LIFE randomized trial. JAMA 314, 781-790. 
[7] Ohman H, Savikko N, Strandberg TE, Pitkälä KH (2014) Effect of physical exercise on cognitive performance in older adults with mild cognitive impairment or dementia: A systematic review. Dement Geriatr Cogn Disord 38, 347-365.

[8] Baker LD, Frank LL, Foster-Schubert K, Green PS, Wilkinson CW, McTiernan A, Plymate SR, Fishel MA, Watson GS, Cholerton BA, Duncan GE, Mehta PD, Craft S (2010) Effects of aerobic exercise on mild cognitive impairment: A controlled trial. Arch Neurol 67, 71-79.

[9] Forbes D, Thiessen EJ, Blake CM, Forbes SC, Forbes S (2013) Exercise programs for people with dementia. Cochrane Database Syst Rev 12, CD006489.

[10] Lautenschlager NT, Cox KL, Flicker L, Foster JK, van Bockxmeer FM, Xiao J, Greenop KR, Almeida OP (2008) Effect of physical activity on cognitive function in older adults at risk for Alzheimer disease: A randomized trial. JAMA 300, 1027-1037.

[11] Vreugdenhil A, Cannell J, Davies A, Razay G (2012) A community-based exercise programme to improve functional ability in people with Alzheimer's disease: A randomized controlled trial. Scand J Caring Sci 26, 12-19.

[12] Rocca P, Leotta D, Liffredo C, Mingrone C, Sigaudo M, Capellero B, Rocca G, Simoncini M, Pirfo E, Bogetto F (2010) Neuropsychiatric symptoms underlying caregiver stress and insight in Alzheimer's disease. Dement Geriatr Cogn Disord 30, 57-63.

[13] Frederiksen KS, Sobol N, Beyer N, Hasselbalch S, Waldemar G (2014) Moderate-to-high intensity aerobic exercise in patients with mild to moderate Alzheimer's disease: A pilot study. Int J Geriatr Psychiatry 29, 1242-1248.

[14] Craig P, Dieppe P, Macintyre S, Michie S, Nazareth I, Petticrew M (2008) Developing and evaluating complex interventions: The new Medical Research Council guidance. BMJ 337, a1655.

[15] Hoffmann K, Frederiksen KS, Sobol NA, Beyer N, Vogel A, Simonsen AH, Johannsen P, Lolk A, Terkelsen O, Cotman CW, Hasselbalch SG, Waldemar G (2013) Preserving cognition, quality of life, physical health and functional ability in Alzheimer's disease: The effect of physical exercise (ADEX trial): Rationale and design. Neuroepidemiology 41, 198-207.

[16] McKhann G, Drachman D, Folstein M, Katzman R, Price D, Stadlan EM (1984) Clinical diagnosis of Alzheimer's disease: Report of the NINCDS-ADRDA Work Group under the auspices of Department of Health and Human Services Task Force on Alzheimer's Disease. Neurology 34, 939-944.

[17] Cink RE, Thomas TR (1981) Validity of the Astrand-Ryhming nomogram for predicting maximal oxygen intake. Br J Sports Med 15, 182-185.

[18] Smith A (1982) Symbol Digit Modalities Test (SDMT) Manual (revised 1982). Western Psychological Services, Los Angeles.

[19] Rosen WG, Mohs RC, Davis KL (1984) A new rating scale for Alzheimer's disease. Am J Psychiatry 141, 1356-1364.

[20] Golden C. (1978) Stroop color and word test manual. Stoelting Co., Chicago.

[21] Folstein MF, Folstein SE, McHugh PR (1975) Mini-mental state. A practical method for grading the cognitive state of patients for the clinician. J Psychiatr Res 12, 189-198.

[22] Hamilton M (1960) A rating scale for depression. J Neurol Neurosurg Psychiatry 23, 56-62.

[23] Galasko D, Bennett D, Sano M, Ernesto C, Thomas R, Grundman M, Ferris S (1997) An inventory to assess activities of daily living for clinical trials in Alzheimer's disease. The Alzheimer's Disease Cooperative Study. Alzheimer Dis Assoc Disord 11(Suppl 2), S33-S39.
[24] Cummings JL, Mega M, Gray K, Rosenberg-Thompson S, Carusi DA, Gornbein J (1994) The Neuropsychiatric Inventory: Comprehensive assessment of psychopathology in dementia. Neurology 44, 2308-2314.

[25] The EuroQol, Group (1990) EuroQol-a new facility for the measurement of health-related quality of life. Health Policy 16, 199-208.

[26] EQ-5D-5L Value Sets. http://www.euroqol.org/about-eq-5d/ valuation-of-eq-5d/eq-5d-51-value-sets.html. Assessed June $16,2015$.

[27] Dufouil C, Brayne C, Clayton D (2004) Analysis of longitudinal studies with death and drop-out: A case study. Stat Med 23, 2215-2226.

[28] Benjamini Y, Hochberg Y (1995) Controlling the false discovery rate: A practical and powerful approach to multiple testing. J R Stat Soc Series B Stat Methodol 57, 289-300.

[29] Osterberg L, Blaschke T (2005) Adherence to medication. $N$ Engl J Med 353, 487-497.

[30] Yu F, Swartwood RM (2012) Feasibility and perception of the impact from aerobic exercise in older adults with Alzheimer's disease. Am J Alzheimers Dis Other Demen 27, 397-405.

[31] Nascimento CM, Teixeira CV, Gobbi LT, Gobbi S, Stella F (2012) A controlled clinical trial on the effects of exercise on neuropsychiatric disorders and instrumental activities in women with Alzheimer's disease. Rev Bras Fisioter 16, 197-204.

[32] Stevens J, Killeen M (2006) A randomised controlled trial testing the impact of exercise on cognitive symptoms and disability of residents with dementia. Contemp Nurse 21, 32-40.

[33] Coelho FG, Vital TM, Stein AM, Arantes FJ, Rueda AV, Camarini R, Teodorov E, Santos-Galduróz RF (2014) Acute aerobic exercise increases brain-derived neurotrophic factor levels in elderly with Alzheimer's disease. J Alzheimers Dis 39, 401-408.

[34] Nascimento CM, Pereira JR, de Andrade LP, Garuffi M, Talib LL, Forlenza OV, Cancela JM, Cominetti MR, Stella F (2014) Physical exercise in MCI elderly promotes reduction of proinflammatory cytokines and improvements on cognition and BDNF peripheral levels. Curr Alzheimer Res 11, 799-805.

[35] Bennett DA, Wilson RS, Schneider JA (2012) Natural history of mild cognitive impairment in older persons. Neurology $\mathbf{5 9}$, 198-205.

[36] Wilbur J, Marquez DX, Fogg L, Wilson RS, Staffileno BA, Hoyem RL, Morris MC, Bustamante EE, Manning AF (2012) The relationship between physical activity and cognition in older Latinos. J Gerontol B Psychol Sci Soc Sci 67, 525-534.

[37] Netz Y, Wu MJ, Becker BJ, Tenenbaum G (2005) Physical activity and psychological well-being in advanced age: A meta-analysis of intervention studies. Psychol Aging 20, 272-284.

[38] Van de Winckel A, Feys H, De Weerdt W, Dom R (2004) Cognitive and behavioural effects of music-based exercises in patients with dementia. Clin Rehabil 18, 253-260.

[39] Kemoun G, Thibaud M, Roumagne N, Carette P, Albinet C, Toussaint L, Paccalin M, Dugué B (2010) Effects of a physical training programme on cognitive function and walking efficiency in elderly persons with dementia. Dement Geriatr Cogn Disord 29, 109-114.

[40] Nelson ME, Rejeski WJ, Blair SN, Duncan PW, Judge JO, King AC, Macera CA, Castaneda-Sceppa C (2007) Physical activity and public health in older adults: Recommendation from the American College of Sports Medicine and the American Heart Association. Circulation 116, 1094-1105. 
[41] Kirk-Sanchez NJ, McGough EL (2009) Physical exercise and cognitive performance in the elderly: Current perspectives. Clin Interv Aging 9, 51-62.

[42] Washburn RA, Smith KW, Jette AM, Janney CA (1993) The Physical Activity Scale for the Elderly (PASE): Development and evaluation. J Clin Epidemiol 46, 153-162.

[43] Liu-Ambrose T, Nagamatsu LS, Graf P (2010) Resistance training and executive functions: A 12-month randomized controlled trial. Arch Intern Med 170, 170-178.

[44] Olazaran J, Reisberg B, Clare L, Cruz I, Peña-Casanova J, Del Ser T, Woods B, Beck C, Auer S, Lai C, Spector A, Fazio S, Bond J, Kivipelto M, Brodaty H, Rojo JM, Collins H, Teri L, Mittelman M, Orrell M, Feldman HH, Muñiz R (2010)
Nonpharmacological therapies in Alzheimer's disease: A systematic review of efficacy. Dement Geriatr Cogn Disord 30, 161-178.

[45] Leong C (2014) Antidepressants for depression in patients with dementia: A review of the literature. Consult Pharm 29, 254-263.

[46] Seitz DP, Gill SS, Herrmann N (2013) Pharmacological treatments for neuropsychiatric symptoms of dementia in long-term care: A systematic review. Int Psychogeriatr 25, 185-203.

[47] Yaffe K, Fox P, Newcomer R (2002) Patient and caregiver characteristics and nursing home placement in patients with dementia. JAMA 287, 2090-2097. 